\title{
Coherent Excitation-Selective Spectroscopy in Planar Metamaterials
}

\author{
Xu Fang, ${ }^{1}$ Ming Lun Tseng, ${ }^{2,3}$ Din Ping Tsai, ${ }^{2,3,4}$ and Nikolay I. Zheludev ${ }^{1,5}$ \\ ${ }^{1}$ Optoelectronics Research Centre and Centre for Photonic Metamaterials, University of Southampton, Southampton SO17 1BJ, UK \\ ${ }^{2}$ Graduate Institute of Applied Physics, National Taiwan University, Taipei 106, Taiwan \\ ${ }^{3}$ Department of Physics, National Taiwan University, Taipei 106, Taiwan \\ ${ }^{4}$ Research Center for Applied Sciences, Academia Sinica, Taipei 115, Taiwan \\ ${ }^{5}$ Centre for Disruptive Photonic Technologies, Nanyang Technological University, Singapore 637371, Singapore \\ x.fang@soton.ac.uk;niz@orc.soton.ac.uk
}

\begin{abstract}
We demonstrated that the electric and magnetic resonances of metamaterials can be separately switches off and on by positioning the metamaterials along a standing wave, while both resonances are present in travelling-wave spectra.

OCIS codes: (160.3918) Metamaterials; (300.0300) Spectroscopy
\end{abstract}

We demonstrate an excitation-selective spectroscopy, in which the optical transitions of different nature in nanoscale thick films can be manipulated by using a standing wave formed by two counter-propagating coherent waves. By placing the thin films at the electric or magnetic antinodes of the standing wave, one can selectively emphasize the electric or the magnetic dipole transitions correspondingly. In a proof-of-principle experiment with periodic arrays of slit nano-antennas and "magnetic wire" metamaterials exhibiting respectively electric dipolar and magnetic dipolar resonances, we demonstrated that the electric and magnetic resonances can be separately switches off and on by positioning the metamaterials along a standing wave, while both resonances are present in travelling-wave spectra. Our demonstration could be generalized to spectroscopic application when a new degree of freedom, the position in the standing wave, is used for the purpose of excitation-selective spectroscopy in sub-wavelength thick films.

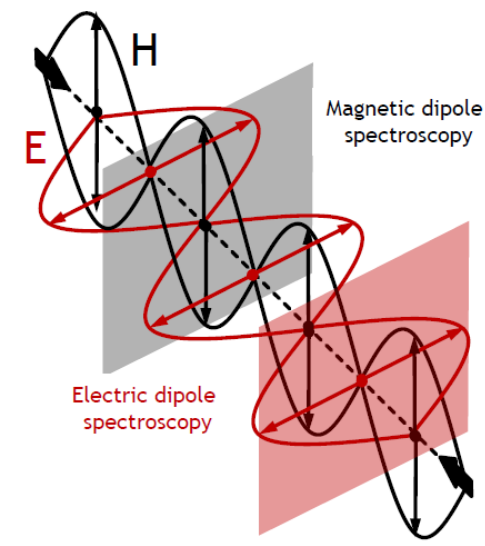

Fig. 1. Schematic of coherent excitation-selective spectroscopy.

The origin of the excitation-selective spectroscopy in the field of a standing wave (Fig. 1) can be understood by considering $H_{\text {int }}$, the Hamiltonian of interaction between electromagnetic radiation and matter, which can written as

$$
H_{\text {int }}=\frac{1}{c} \widehat{\boldsymbol{d}} \frac{\partial \boldsymbol{A}}{\partial t}-\frac{1}{c}\left(\frac{d \hat{q}^{i j}}{d t}-c e_{i j k} \widehat{\boldsymbol{m}}^{k}\right) \nabla_{j} \boldsymbol{A}_{i}
$$

where $\boldsymbol{A}$ is the vector-potential of the electromagnetic field, and $\widehat{\boldsymbol{d}}, \widehat{\boldsymbol{q}}$, and $\widehat{\boldsymbol{m}}$ are operators of electric dipole, electric quadrupole and magnetic dipole of the medium, respectively [1]. If two linearly polarized coherent counter-propagating waves $\boldsymbol{A}_{x}=A_{0} \cos (\omega t-k z)+A_{0} \cos (\omega t+k z)$ oscillating at frequency $\omega$ form a standing wave along $\mathrm{z}$-direction, the time dependent Hamiltonian of interaction can be reduced to

$$
H_{\text {int }}=-2 A_{0} k\left[\widehat{\boldsymbol{d}}^{x} \sin (\omega t) \cos (k z)-\left(\widehat{\boldsymbol{m}}^{y}+\frac{1}{c} \frac{d \widehat{\boldsymbol{q}}^{x z}}{d t}\right) \cos (\omega t) \sin (k z)\right]
$$

Hence, if a subwavelength thick layer of matter is placed at the electric nodes of the wave where $\cos (k z)=0$, the electric dipole interaction vanishes while magnetic dipole and electric quadrupole interactions become the dominant terms of the Hamiltonian. On the contrary, if the layer is placed at the magnetic nodes where $\sin (k z)=0$, the magnetic dipole and the electric quadrupole interactions vanish while the electric dipole interaction becomes the dominant term of the interaction. 

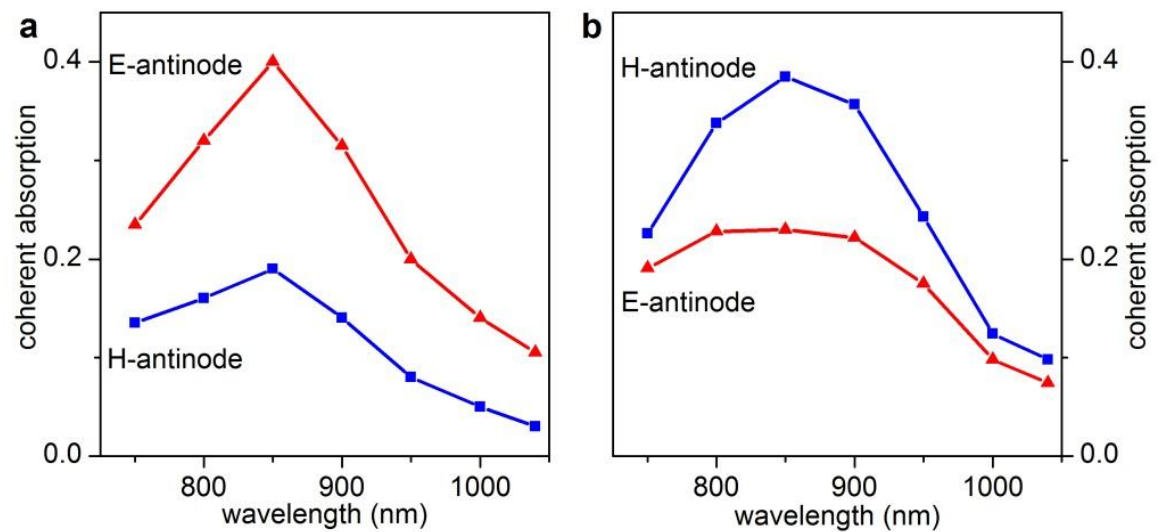

Fig. 2. Excitation-selective spectra of two types of metamaterials. (a) A planar array of slit nano-antennas working as electric dipoles. (b) A planar array of nanowires working as magnetic dipoles.

We have experimentally demonstrated coherent excitation-selective spectroscopy in two types of metamaterials. In our experiments we used a Ti:sapphire tunable laser to characterize travelling wave and standing wave absorption using an interferometry arrangement [2].

In the first case the metamaterial is a planar array of slit nano-antennas (Fig. 2a), the optical response of which is dominated by an electric dipolar resonance. The metamaterial is fabricated from a $A u / \mathrm{Si}_{3} \mathrm{~N}_{4}$ film using focused ion beam (FIB) milling. The thickness of the whole structure is $80 \mathrm{~nm}$. An absorption peak at approximately $870 \mathrm{~nm}$ is observed in its travelling-wave spectrum, which is associated with the electric resonance arising from the dipolar surface charge oscillation of the nano-antennas. Figure 2a shows absorption spectra of the metamaterial in a standing wave. At the electric antinode (E-antinode), the absorption peak at 870 $\mathrm{nm}$ is almost twice as strong as that of the travelling-wave. No substantial absorption is seen at the magnetic antinode (H-antinode).

In the second case, the metamateraisl is a three-layered structure, the optical response of which is dominated by the magnetic dipolar resonance (Fig. 2b). The metamaterial is fabricated from a Au/Si${ }_{3} \mathrm{~N}_{4} / \mathrm{Au}$ film using FIB milling. The thickness of the whole structure is $110 \mathrm{~nm}$. When the metamaterial is placed at the magnetic antinode of the standing wave, it exhibits strong absorption at $890 \mathrm{~nm}$. In comparison, the absorption at the electric antinode is significantly lower.

Our demonstration could be generalized to spectroscopic application when a new degree of freedom, the position in the standing wave, is used. This excitation-selective spectroscopy will facilitate detection of weak resonances in sub-wavelength thick films.

[1] Yu. P. Svirko and N. I. Zheludev, Polarization of Light in Nonlinear Optics (Wiley, Chichester, 1998), p. 182.

[2] J. Zhang, K. F. MacDonald \& N. I. Zheludev. "Controlling light-with-light without nonlinearity,” Light: Sci. Appl 1, e18 (2012). 